INFNFE-01-99

\title{
Helioseismology and Beryllium neutrinos
}

\author{
B. Ricci ${ }^{1}$, F. L. Villante ${ }^{1}$ and M. Lissia ${ }^{2}$, \\ ${ }^{1}$ Dipartimento di Fisica dell'Università di Ferrara and Istituto Nazionale di Fisica Nucleare, \\ Sezione di Ferrara, via Paradiso 12, I-44100 Ferrara, Italy . \\ ${ }^{2}$ Istituto Nazionale di Fisica Nucleare, Sezione di Cagliari and Dipartimento di Fisica, Università \\ di Cagliari, Cittadella Universitaria, I-09042 Monserrato, Italy.
}

(April 1999)

\begin{abstract}
We derive a lower limit on the Beryllium neutrino flux on earth, $\Phi(B e)_{\min }=$ $1 \cdot 10^{9} \mathrm{~cm}^{-2} \mathrm{~s}^{-1}$, in the absence of oscillations, by using helioseismic data, the B-neutrino flux measured by Superkamiokande and the hydrogen abundance at the solar center predicted by Standard Solar Model (SSM) calculations. We emphasize that this abundance is the only result of SSMs needed for getting $\Phi(B e)_{\min }$. We also derive lower bounds for the Gallium signal, $G_{\min }=$ $(91 \pm 3) \mathrm{SNU}$, and for the Chlorine signal, $C_{\min }=(3.24 \pm 0.14) \mathrm{SNU}$, which are about $3 \sigma$ above their corresponding experimental values, $G_{\text {exp }}=(72 \pm 6)$ $\mathrm{SNU}$ and $C_{\text {exp }}=(2.56 \pm 0.22) \mathrm{SNU}$.
\end{abstract}

\section{INTRODUCTION}

There are several indications, but not really a proof of solar neutrino oscillations (see e.g. [1,2]):

i) the result of all five solar neutrino experiments are below the predictions of SSM calculations;

ii) unless neutrino oscillate, the experimental results for Gallium, Chlorine and water detectors are hardly consistent among themselves, even if some experimental result is discarded; iii) the measured Gallium signal, $G_{\exp }=(72 \pm 6) \mathrm{SNU}$, is below the minimal value expected by the luminosity constraint, $G_{\text {Lum }}=(79 \pm 2)$ SNU, for standard neutrinos.

Future experiments like Borexino [3] and LENS [4], aiming at the detection of Beneutrinos from

$$
{ }^{7} \mathrm{Be}+e^{-} \rightarrow{ }^{7} \mathrm{Li}+\nu_{e}
$$

can be crucial for the solar neutrino puzzle, since oscillation schemes predict unambiguous seasonal modulations and/or drastic reductions with respect to the predicted flux [5]:

$$
\Phi(B e)_{S S M}=4.8 \cdot(1 \pm 0.09) \cdot 10^{9} \mathrm{~cm}^{-2} \mathrm{~s}^{-1} .
$$


This flux is a very robust prediction of SSM calculation, see [5] for a discussion. The quoted $1 \sigma$ error is dominated by uncertainty on the ${ }^{3} \mathrm{He}+{ }^{4} \mathrm{He}$ cross section, as emphasized in [5] and in [6]. This uncertainty alone contributes as much as all astrophysical uncertainties (metal abundance, opacity, luminosity, diffusion...) combined together.

Nevertheless, eq. (2) represents the outcome of an involved solar model calculation, and it is useful to provide a lower limit in the absence of oscillations, $\Phi(B e)_{\text {min }}$, which is essentially independent of the SSM. Such is the aim of the present note.

As well known, Be-neutrinos give a significant contribution to the Gallium and Chlorine signals, being respectively about one fourth and one sixth of the total, according again to the SSM. The lower limit on $\Phi(B e)$ can thus be used to determine lower limits to the Gallium and Chlorine signal, in the absence of oscillations.

\section{A LOWER LIMIT TO THE PRODUCTION RATE OF BE-NEUTRINOS}

The B-neutrinos from

$$
\begin{aligned}
{ }^{7} B e+p \rightarrow & { }^{8} B+\gamma \\
& { }^{8} B \rightarrow 2 \alpha+e^{+}+\nu_{e},
\end{aligned}
$$

have been observed by Kamiokande [7] and Superkamiokande [8]. Since B-neutrinos and Be-neutrinos are both sons of ${ }^{7} \mathrm{Be}$ nuclei, one expects that detection of the former gives information on the latter. Our aim is to determine a lower limit on the production rate of Be-neutrinos, $L(B e)$, starting from this consideration.

As the rates of (11) and (3) depend differently on the solar temperature, we need some information on it. This is (indirectly) provided by helioseismology, which determines the sound speed with an accuracy of one per cent or better, even close to the solar center, see e.g. [9]. Temperature is obtained from the sound speed if the chemical composition of the solar plasma is known. This is the only information which we shall take from SSMs, in the form of the hydrogen abundance at the solar centre $X_{c}$, a quantity which is largely independent of solar models, since it reflects the amount of hydrogen burnt all along the sun history. Let us make this argument in some detail.

The ${ }^{7} B e$ and ${ }^{8} B$ luminosities, at production, can be written as:

$$
\begin{aligned}
L(B) & =\int d^{3} r n_{1} n_{7}<\sigma v>_{17}=\lambda_{17} / T_{o}^{13} \int d^{3} r n_{1} n_{7} T^{13} \\
L(B e) & =\int d^{3} r n_{e} n_{7}<\sigma v>_{e 7}=\lambda_{e 7} / T_{o}^{-0.5} \int d^{3} r n_{e} n_{7} T^{-0.5}
\end{aligned}
$$

where we have used a parametrization of the form $\langle\sigma v\rangle_{i j}=\lambda_{i j}\left(T / T_{o}\right)^{\alpha_{i j}}$, the temperature scale $T_{o}$ is chosen as the central temperature of the model in [5], hereafter BP98, $T_{o}=$ $1.5697 \cdot 10^{7} \mathrm{~K}$, and according to [10]:

$$
\begin{aligned}
& \lambda_{e 7}=2.34 \cdot 10^{-33}(1 \pm 2 \%) \mathrm{cm}^{3} \mathrm{~s}^{-1} \\
& \lambda_{17}=1.04 \cdot 10^{-35}(1 \pm 16 \%) \mathrm{cm}^{3} \mathrm{~s}^{-1}
\end{aligned}
$$

(here and in the following errors shown are combinations in quadrature of systematic and statistical $1 \sigma$ errors). 
By using eqs. (4) and (5) one can relate the production rates of Boron and Beryllium neutrinos. From eq. (雨) one has:

$$
L(B)=\frac{\lambda_{17}}{\lambda_{e 7}} T_{o}^{-13.5} \int d^{3} r n_{e} n_{7}<\sigma v>_{e 7} T^{13.5} x
$$

where $x=n_{1} / n_{e}$ is the ratio of free protons to electrons. Since temperature decreases sharply when moving away from the solar center, one can assume that at any point in the solar interior $x(r) T(r)^{13.5} \leq x_{c} T_{c}^{13.5}$, where, here and in the following, the suffix $c$ refers to the solar center. In this way one has:

$$
L(B) \leq \frac{\lambda_{17}}{\lambda_{e 7}}\left(\frac{T_{c}}{T_{o}}\right)^{13.5} x_{c} L(B e) .
$$

As the produced $\nu_{e}$ can oscillate into species with a smaller or vanishing cross section in the detector, the observed luminosity $L(B)_{o b s}$ in Kamiokande and Superkamiokande cannot exceed the produced luminosity, $L(B)_{o b s} \leq L(B)$, so that one has the following lower limit for $L_{B e}$ :

$$
L(B e) \geq L(B)_{o b s} \frac{\lambda_{e 7}}{\lambda_{17}}\left(\frac{T_{c}}{T_{o}}\right)^{13.5} \frac{1}{x_{c}} .
$$

Now we use the fact that the solar center can be described as perfect gas of fully ionized $\mathrm{H}$ and $\mathrm{He}$, to a very good approximation. In terms of the isothermal squared sound speed $u=P / \rho$ and of the hydrogen mass fraction $X$, this gives: $k T_{c}=u_{c} m_{p} /\left(3 / 4+5 / 4 X_{c}\right)$. One also has: $x_{c}=2 X_{c} /\left(X_{c}+1\right)$. In this way one gets:

$$
L(B e) \geq \frac{\lambda_{e 7}}{\lambda_{17}}\left(\frac{k T_{o}}{m_{p} u_{c}}\right)^{13.5} \frac{\left(X_{c}+1\right)}{\left(2 X_{c}\right)}\left(5 / 4 X_{c}+3 / 4\right)^{13.5} L(B)_{o b s}
$$

The equation above can of course be translated in terms of fluxes.

We take $\Phi(B)_{o b s}=2.42 \cdot(1 \pm 3 \%) \cdot 10^{6} \mathrm{~cm}^{-2} \mathrm{~s}^{-1}$ from [8] and $u_{c}=1.53 \cdot(1 \pm 1 \%) \cdot 10^{15} \mathrm{~cm}^{2} / \mathrm{s}^{2}$, in agreement with helioseismic determinations, see [9].

For the central hydrogen abundance we have to rely on solar model calculations. Recent SSM calculations all yield $X_{c}$ in the narrow range $0.333<X_{c}<0.347$, with a mean value close to the BP98 estimate, $X_{c}^{B P 98}=0.339$, see table凹. The calculated value of $X_{c}$ is sensitive

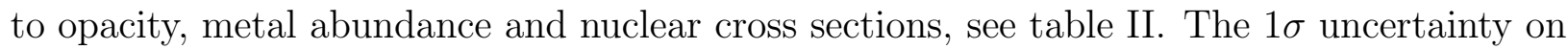
opacity and metal abundance are respectively $5 \%$ and $6 \%$, according to [2] and [11], and that on $S_{p p}$ is $1.7 \%$, from [10]. By computing suitable solar models and adding errors in quadrature, we conclude:

$$
X_{c}=0.339 \pm 0.010
$$

We have also computed $X_{c}$ for a series of "non standard solar models", where some input parameters have been varied, one at the time, by about $\pm 3 \sigma$ with respect to the SSM reference input, see table $\mathbb{1}$. Even in this case, $X_{c}$ stays in the range $0.329-0.358$, i.e. within about $\pm 5 \%$ from BP98. 
We remark that $X_{c}$ is essentially an indicator of how much hydrogen has been burnt so far, starting from an initial value $X_{i n}$ about 0.7 . The stability of $X_{c}$ corresponds to the fact that any solar model has to account for an integrated solar luminosity of about $L_{\odot} t_{\odot}=5.5 \cdot 10^{50} \mathrm{erg}$. On these grounds, we consider the adopted value of $X_{c}$ as rather safe.

In this way we get:

$$
\Phi(B e) \geq(1 \pm 0.24) \cdot 10^{9} \mathrm{~cm}^{-2} \mathrm{~s}^{-1} .
$$

where the error include, in quadrature, all uncertainties mentioned above. The uncertainty on $\lambda_{17}, X_{c}$ and $u_{c}$ contribute to the total error $16 \%, 13 \%$ and $12 \%$ respectively.

The inequality (13) defines a minimum flux $\Phi(B e)_{\text {min }} \simeq 1 \cdot 10^{9} \mathrm{~cm}^{-2} \mathrm{~s}^{-1}$, which is one fifth of the SSM prediction, see eq. (2). We note that the only input from SSM is the value of $X_{c}$, whereas all other inputs, $\Phi(B)_{\text {obs }}$ and $u_{c}$, are from observational data.

We have obtained this minimal flux using only the physical information that is relevant to the ratio between the Beryllium and Boron luminosities $L(B e) / L(B)$; for instance, the actual value of $n_{7}(r)$ never matters for our result. However, the additional physical information that determines the two fluxes separately, in particular the measured $\Phi(B)$, can only strengthen this limit. In fact, a solar-model-independent analysis of the Beryllium and Boron neutrino flux production $\left(0<T_{c}<\infty\right.$, cross sections more than $3 \sigma$ 's away from the central values, profiles of densities that are not constrained by helioseismology varied by factors larger than 30 ) shows that the lower limit to $\Phi(B e)$ is $1.6 \times 10^{9} \mathrm{~cm}^{-2} \mathrm{~s}^{-1}$, as can be inferred from Fig. 3 of Ref. 12 and the measured $\Phi(B)$.

\section{IMPLICATIONS FOR SOLAR NEUTRINO EXPERIMENTS}

\section{A. Borexino and LENS}

The relevance of the bound (13) can be appreciated when discussing the complementarity between Borexino and LENS. We remind that LENS is sensitive to $\nu_{e}$ only, whereas the signal of Borexino can get contribution also from $\nu_{\mu}$ or $\nu_{\tau}$, their cross section being about $1 / 5$ than that of $\nu_{e}$.

For both experiments, a signal well below $1 / 5$ of the SSM prediction will be a definite proof of neutrino oscillations, since it leads to a violation of eq. (13).

A signal at the level of $1 / 5$ of the SSM prediction in Borexino could be interpreted as due to Small Mixing (SM) angle oscillations into active neutrinos, where one expects that all $\nu_{e}$ from Beryllium have been transformed into $\nu_{\mu}$. However, one could still insist on standard neutrinos, arguing for some drastic (maybe desperate) modification of the solar model. In this situation, a clear discrimination will be provided by LENS: for the SM case, the LENS signal, barrying the background, has to vanish, so that the bound (13) will be violated, giving a definite proof of neutrino oscillations (furthermore, the comparison with Borexino will show the presence of $\nu_{\mu}$ or $\nu_{\tau}$ ). 


\section{B. Gallium experiments}

As well known, the solar luminosity essentially fixes the total production rate of neutrinos. Since neutrino cross sections increase with energy, the minimal Gallium signal, in the absence of oscillations, can be estimated by assuming that the total flux consists of pp neutrinos only. The pep-neutrinos can be safely included in this estimate, as the ratio of pep to pp-neutrinos is well known and essentially unsensitive to solar physics details, see [13] and [2]. By using updated cross sections from [14], this arguments gives as a minimal Gallium signal in the absence of oscillations:

$$
G_{\text {Lum }}=(79.5 \pm 2.0) \mathrm{SNU}
$$

where the error arises mainly from the capture cross section of pp neutrinos.

As well known, also B-neutrinos contribute to the Gallium signal. Their contribution is best estimated by using experimental data. If one takes into account the flux measured by Superkamiokande, with the capture cross section of [14], this contributes an additional $(5.8 \pm 1.5)$ SNU, where most of the error comes again from the capture cross section. All this results in:

$$
G_{L u m+S K}=(85.3 \pm 2.5) \mathrm{SNU}
$$

According to the previous discussion, one has to include now the minimal contribution of ${ }^{7} \mathrm{Be}$ neutrinos. For $\Phi(B e)=\Phi(B e)_{\min }$ by using the luminosity constraint (see section 2.4 of [2]) one has an additional contribution of (5.9 \pm 1.4$)$ SNU, where most of the error comes from $\lambda_{17}$, so that in conclusion the minimal Gallium signal is now:

$$
G_{\min }=(91 \pm 3) \mathrm{SNU} .
$$

This has to be compared with the Gallex [15] and Sage [16] average:

$$
G_{\text {exp }}=(72 \pm 6) \mathrm{SNU} \text {. }
$$

All this means that the present experimental result is about three sigmas below the minimal expectation in the absence of oscillations.

This also illustrates the potential of GNO [17], the successor of Gallex, which should reduce the total error down to about $4 \mathrm{SNU}$. If the present central value is mantained, the discrepancy with the minimal prediction will be at the level of about $5 \sigma$, thus providing a clean signature of neutrino oscillations.

\section{The Chlorine result}

The solar luminosity constraint, together with the assumption that the ratio of pepneutrinos over pp-neutrinos $\psi=\Phi(p e p) / \Phi(p p)=0.0023$ is correctly determined by SSM calculation, can be used to provide a lower limit also for the Chlorine signal.

As well known, due to the fact that cross sections increase with neutrino energy, the minimal signal is obtained by maximixing the number of lowest-energy neutrinos, consistent 
with the luminosity constraint. Since a flux $\Phi_{p p}$ of pp-neutrinos is anyhow accompanied by a flux $\Phi(p e p)=\psi \Phi(p p)$, this implies a minimal Chlorine signal:

$$
C_{L u m}=\frac{K_{\odot}}{Q_{p p} / \psi+Q_{p e p}} \sigma_{p e p}
$$

where $K_{\odot}$ is the solar constant, $Q_{i}$ is the average electromagnetic energy released for emitted $i$-neutrino (see [2]) and $\sigma_{i}$ is the averaged $i$-neutrino cross section on Chlorine detector. By using the cross section from 18], but for the absorption cross section of ${ }^{8} B$ neutrinos from [19], one has:

$$
C_{\text {Lum }}=(0.243 \pm 0.005) \mathrm{SNU} .
$$

From the Superkamiokande result one can deduce the B-neutrinos contribution of $(2.76 \pm$ 0.12) SNU, so that:

$$
C_{\text {Lum }+S K}=(3.0 \pm 0.1) \mathrm{SNU} .
$$

The minimal Be-neutrino flux implies the additional contribution of

$$
\begin{aligned}
C_{B e_{\min }} & =\Phi(B e)_{\min }\left[\sigma_{B e}-\sigma_{p e p} \frac{Q_{B e}}{Q_{p p} / \psi+Q_{p e p}}\right] \\
& =(0.24 \pm 0.06) \mathrm{SNU} .
\end{aligned}
$$

In this way the minimal Chlorine signal becomes:

$$
C_{\text {min }}=(3.24 \pm 0.14) \mathrm{SNU}
$$

to be compared with the experimental result 20]

$$
C_{\text {exp }}=(2.56 \pm 0.22) \mathrm{SNU}
$$

Again the Be-neutrinos contributions, eq. (21), corresponds to the " $1 \sigma$ " uncertainty of the experimental result, and again the experimental signal is about three sigmas below the minimal prediction.

\section{Combining experimental results}

A global view of the "solar neutrino puzzle" is presented in Fig. 目 which updates Fig. 7 of [2]. As a generalization of eq. (13) for an arbitrary value of the observed ${ }^{8} B$ flux $\Phi(B)$ one has:

$$
\Phi(B e)_{\min }=4 \cdot 10^{2} \Phi(B) .
$$

The corresponding thick "diagonal" line, in Fig. 1 defines thus the lower border of the physical region. The shaded area, corresponding to the region within $3 \sigma$ from each experimental result, is almost completely out of the physical region. 


\section{CONCLUSIONS}

As a summary, in the absence of oscillations we predict:

$$
\begin{aligned}
\Phi(B e)_{\min } & =(1 \pm 0.24) \cdot 10^{9} \mathrm{~cm}^{-2} \mathrm{~s}^{-1} \\
G_{\min } & =(91 \pm 3) \mathrm{SNU} \\
C_{\min } & =(3.24 \pm 0.14) \mathrm{SNU}
\end{aligned}
$$

Let us list the information and assumptions behind these results:

i) the measured ${ }^{8} B$ flux by Superkamiokande;

ii) the helioseismically determined sound speed, near or at the solar center, $u_{c}$;

iii) the measured value of $\lambda_{17}$;

iv) the value of $\lambda_{e 7}$, derived from the lifetime of ${ }^{7} B e$ in the laboratory;

v) the luminosity constraint, i.e. the present observed solar luminosity equals the presently generated nuclear power in the sun;

v) the central Hydrogen abundance, the only information we take from SSM calculations.

We remark that we do not need to know the central solar temperature, nor the values of the astrophysical S-factors for the $\mathrm{He}+\mathrm{He}$ reactions.

\section{ACKNOWLEDGMENTS}

We are extremely grateful to V. Berezinsky and G. Fiorentini for suggesting us the problem and for useful discussions and comments. 


\section{REFERENCES}

[1] J. N. Bahcall, P. I. Krastev, and A. Yu. Smirnov, Phys. Rev. D 58 (1998) 096016-1.

[2] V. Castellani, S. Degl'Innocenti, G. Fiorentini, M. Lissia and B. Ricci, Phy. Rep. 281 (1997) 309;

G. Fiorentini and B. Ricci, in Proceedings of Int. Workshop "Beyond the Standard Model:from theory to experiment", Valencia, Spain, October 1997, World Scientific Publishing Co. Settembre 1998.

[3] Borexino collaboration, C. Arpesella et al., "Borexino at Gran Sasso: proposal for a real time detector for low energy solar neutrinos", internal report INFN, Milano, 1992.

[4] R.S. Raghavan, Phys. Rev. Lett. 78 (1997) 3618.

[5] J.N. Bahcall, Sarbani Basu, and M. H. Pinsonneault, Phys. Lett. B 433 (1998), 1.

[6] V. Castellani, S. Degl'Innocenti, W.A. Dziembowski, G. Fiorentini and B. Ricctrino fluxes", Nucl. Phys. B (Proc. Suppl.) 70 (1999) 301 (TAUP97).

[7] Kamiokande coll., Phys. Rev. Lett. 77 (1996) 1683.

[8] Superkamiokande collaboration, talk presented at "Neutrino Telescopes 99", Venice, February 1999, to appear in the proceedings.

[9] S. Degl'Innocenti, W. Dziembowski, G. Fiorentini and B. Ricci, Astr. Phys. 7 (1997) 77.

[10] E.G. Adelberger et al., Rev. Mod. Phys. 70 (1998) 1265.

[11] J.N. Bahcall and M.H. Pinsonneault, with an Appendix on the Age of the Sun by G. J. Wasserburg, Rev. of Mod. Phys. 67 (1995) 781.

[12] V. Berezinsky, G. Fiorentini and M. Lissia, astro-ph/9902222

[13] J.N. Bahcall, B.T. Cleveland, R. Davis Jr. and J. Rowley, ApJ. 292 (1985) L79.

[14] J.N. Bahcall, Phys. Rev. C, 56 (1997) 3391

[15] GALLEX collaboration, XVII International Conference on Neutrino Physics and Astrophysics (Neutrino '98), Takayama, June 1998, Japan, to appear on Nucl. Phys. B (Proc. Suppl.).

[16] SAGE collaboration, XVII International Conference on Neutrino Physics and Astrophysics (Neutrino '98), Takayama, June 1998, Japan, to appear on Nucl. Phys. B (Proc. Suppl.).

[17] Bellotti et al., "Proposal for a permanent Gallium Neutrino Observatory (GNO) at LNGS", LNGS annual report INFN/AE-96/27, pag. 41- 84, http://kosmopc.mpihd.mpg.de/gallex.html;

[18] J.N. Bahcall, "Neutrino Astrophysics", Cambridge University Press, Cambridge 1989.

[19] J.N. Bahcall et al., Phys. Rev. C 54 (1996) 411

[20] B. Cleveland et al., Ap. J. 496 (1998) 505.

[21] O. Richard, S. Vauclair, C. Charbonnel and W.A. Dziembowski, Astr. Astrophys. 312 (1996) 1000.

[22] A.Dar and G. Shaviv, ApJ. 468 (1996) 933.

[23] A.S. Brun, S. Turck-Chieze and P. Morel, Ap. J. 504 (1998) 913.

[24] F. Ciacio, S. Degl'Innocenti and B. Ricci, Astron. and Astrophy. (Suppl. Series) 123 (1997) 449. 


\section{TABLES}

TABLE I. Values of $X_{c}$ in standard and non-standard solar models

\begin{tabular}{|c|c|}
\hline \multicolumn{2}{|c|}{ standard solar models } \\
\hline BP95 [11] & 0.3333 \\
\hline BP98 [5] & 0.3387 \\
\hline RCVD96 [21] & 0.3328 \\
\hline DS96 22 & 0.3424 \\
\hline TC98 23. & 0.3442 \\
\hline FR97 [24] & 0.3467 \\
\hline \multicolumn{2}{|c|}{ non standard solar models } \\
\hline$S_{p p} \times 0.9$ & 0.3414 \\
\hline$S_{p p} \times 1.1$ & 0.3354 \\
\hline opacity $\times 0.9$ & 0.3647 \\
\hline opacity $\times 1.1$ & 0.3287 \\
\hline$Z / X \times 0.9$ & 0.3579 \\
\hline$Z / X \times 1.1$ & 0.3354 \\
\hline
\end{tabular}

TABLE II. Dependence of $X_{c}$ on the solar model inputs $P_{i}$

\begin{tabular}{lc}
\hline \hline$P_{i}$ & $\partial \ln X_{c} / \partial P_{i}$ \\
\hline$S_{p p}$ & 0.1 \\
opacity & -0.5 \\
$Z / X$ & -0.3 \\
\hline \hline
\end{tabular}




\section{FIGURES}

FIG. 1. The ${ }^{8} B$ and ${ }^{7} B e+C N O$ neutrino fluxes, consistent with the luminosity constraint and experimental results for standard neutrinos. The dashed (solid) lines correpond to the central $( \pm 1 \sigma)$ experimental values for Chlorine, Gallium and $\nu-e$ scattering experiments. The dashed area corresponds to the region within $3 \sigma$ from each experimental result. The predictions of solar

models including element diffusion (full circles) [22, 11,21,24], are also shown. The thick diagonal line corresponds to the limit on $\Phi(B e)$ derived in this paper, see eq. (24). 


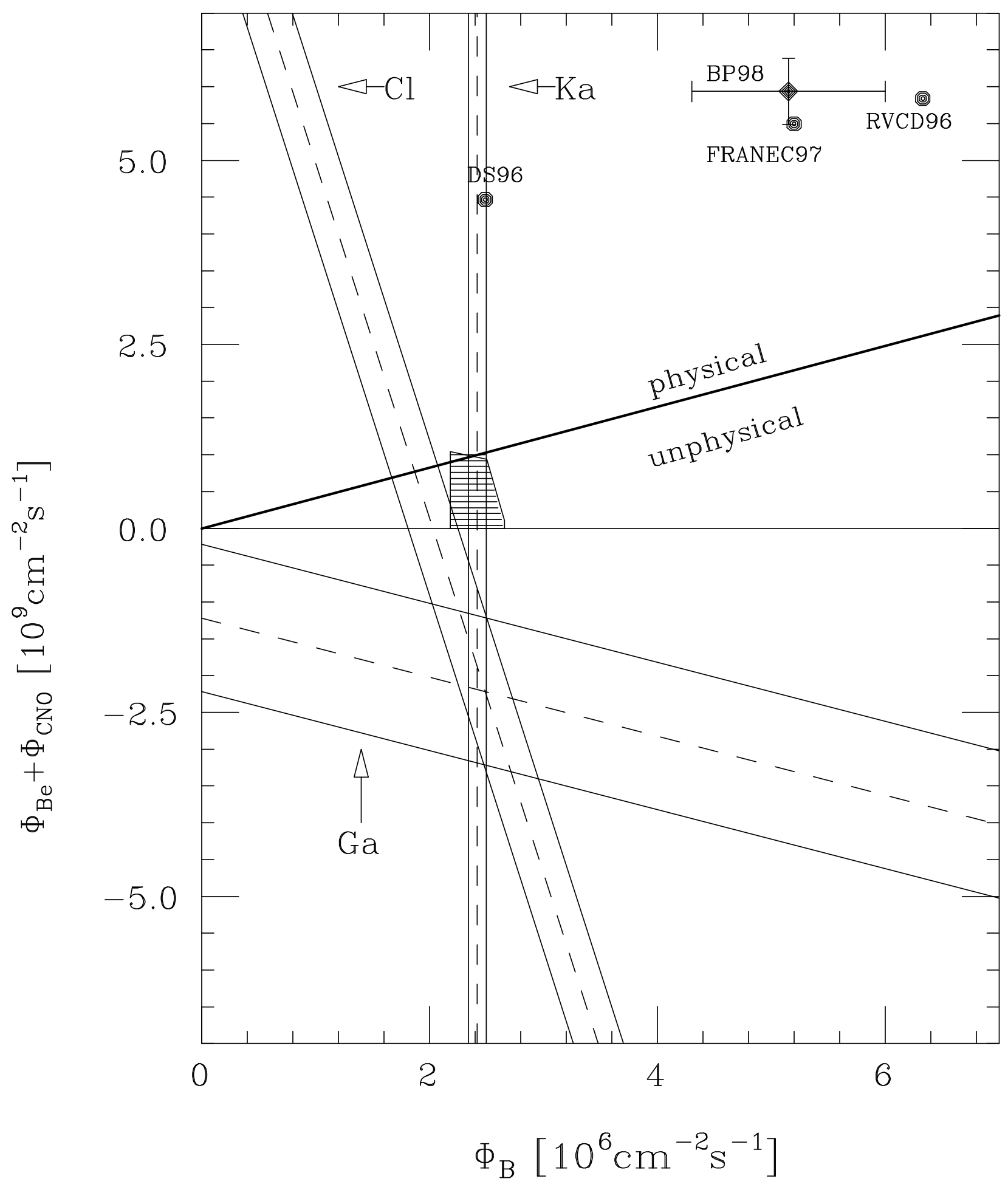

Fig. 1 